\title{
Congenital disorders of glycosylation: a concise chart of glycocalyx dysfunction
}

\author{
Hennet, Thierry ; Cabalzar, Jürg
}

\begin{abstract}
Glycosylation is a ubiquitous modification of lipids and proteins. Despite the essential contribution of glycoconjugates to the viability of all living organisms, diseases of glycosylation in humans have only been identified over the past few decades. The recent development of next-generation DNA sequencing techniques has accelerated the pace of discovery of novel glycosylation defects. The description of multiple mutations across glycosylation pathways not only revealed tremendous diversity in functional impairments, but also pointed to phenotypic similarities, emphasizing the interconnected flow of substrates underlying glycan assembly. The current list of 100 known glycosylation disorders provides an overview of the significance of glycosylation in human development and physiology.
\end{abstract}

DOI: https://doi.org/10.1016/j.tibs.2015.03.002

Posted at the Zurich Open Repository and Archive, University of Zurich ZORA URL: https://doi.org/10.5167/uzh-118114

Journal Article Accepted Version

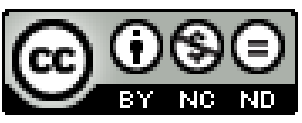

The following work is licensed under a Creative Commons: Attribution-NonCommercial-NoDerivatives 4.0 International (CC BY-NC-ND 4.0) License.

Originally published at:

Hennet, Thierry; Cabalzar, Jürg (2015). Congenital disorders of glycosylation: a concise chart of glycocalyx dysfunction. Trends in Biochemical Sciences, 40(7):377-384.

DOI: https://doi.org/10.1016/j.tibs.2015.03.002 


\title{
Congenital disorders of glycosylation - a consice chart of glycocalyx dysfunction
}

Thierry Hennet, Jürg Cabalzar

Institute of Physiology, University of Zurich, $\mathrm{CH}-8057$ Zurich, Switzerland

\begin{abstract}
Glycosylation is a ubiquitous modification of lipids and proteins. Despite the essential contribution of glycoconjugates to the viability of all living organisms, diseases of glycosylation in humans have only been identified over the past few decades. The recent development of next-generation DNA sequencing techniques has accelerated the pace of discovery of novel glycosylation defects. The description of multiple mutations across glycosylation pathways has revealed a tremendous diversity of functional impairments bus also pointed to phenotypic similarities emphasizing the interconnected flow of substrates underlying glycan assembly. The current list of 100 known glycosylation disorders provides an overview on the significance of glycosylation in human development and physiology.
\end{abstract}

\section{Highlights}

- Congenital disorders underline the role of glycosylation in human development.

- Next-gen sequencing techniques expanded the discovery of glycosylation gene defects.

- The clinical variability of glycosylation disorders implies they are underdiagnosed.

\section{Glycosylation disorders}

Glycosylation is by far the most complex form of protein [1, 2] and lipid modification [3, 4] in all domains of life. The tremendous diversity of glycoconjugate structures resulting from intricate biosynthetic pathways is a major factor hampering the assignment of functions to glycans chains. Much has been learnt from the study of disrupted glycosylation genes in model organisms, thereby establishing numerous essential contributions of glycans in regulating cell and organ functions [5]. The study of human diseases of glycosylation brings additional insights by providing a more differentiated view on glycan functions. Indeed, most human mutations are hypomorphic, thus causing partial loss of glycosylation reactions that lead to variable clinical manifestations.

Diseases of glycosylation are also referred to as congenital disorders of glycosylation (CDG). Given the heterogeneity of glycans, the clinical scope of CDG is considerable, ranging from nearly normal phenotypes to severe multi-organ dysfunctions causing infantile lethality. CDG are rare diseases. The prevalence among CDG types is very different from one type to another, but is largely unknown. The difficulty in identifying patients is another reason behind the rarity of CDG. Unspecific symptoms and the lack of simple laboratory tests make the recognition of CDG cases extremely challenging. The identification of CDG has long relied on the detection of under-glycosylated serum transferrin by 
isoelectric focusing [6]. While easy to perform and requiring only a few microliters of blood, this test exclusively reveals alterations of $\mathrm{N}$-glycosylation. Similar blood tests have unfortunately not been established to reliably diagnose defects in other classes of glycosylation. The simplicity of the serum transferrin test also explains why disorders of $\mathrm{N}$-glycosylation account for the majority of known CDG.

Recent developments in genome-wide DNA sequencing technology enable the identification of mutations without a priori knowledge of candidate genes. As in other fields of biology, nextgeneration sequencing approaches have increased the pace of discovery for new types of CDG [7]. The barrier of 100 genes defects impairing glycosylation has just been passed (see www.physiol.uzh.ch/Glycosylation for a graphical overview). These defects encompass nearly all glycosylation pathways and affect different molecular processes from substrate biosynthesis up to protein trafficking $[8,9]$. The recent application of unbiased strategies such as exome and wholegenome sequencing have further revealed CDG-causing mutations in genes previously not associated with glycosylation, thereby expanding our view on these complex pathways.

CDG have originally been classified in two groups. So-called CDG type-I included defects of lipidlinked oligosaccharide assembly from the formation of dolichol-PP-GIcNAc up to their transfer to asparagine residues on nascent proteins. CDG type-II, by contrast, included defects of $\mathrm{N}$-glycan trimming and elongation as well as defects in any other class of glycosylation [10]. Because several defects affect multiple glycosylation pathways, the artificial distinction between CDG type-I and -II has been replaced by a flat nomenclature simply associating implied genes with the suffix CDG [11]. Functionally, defects can also be grouped based on their contribution to glycosylation reactions (Figure 1). Accordingly, the present review discusses glycosylation disorders through five functional categories, featuring 1) genes encoding glycosyltransferase enzymes, 2) genes involved in donor substrate biosynthesis, 3) genes mediating the translocation of donor substrates, 4) genes regulating glycosyltransferase localization, and 5) genes affecting the homeostasis of secretory organelles.

\section{Glycosyltransferases}

The human genome includes close to 200 glycosyltransferase genes [12]. Glycosyltransferases are the enzymes shaping glycans through the formation of glycosidic linkages. The majority of these glycosyltransferases are transmembrane proteins anchored in the endoplasmic reticulum (ER) and Golgi membranes [13]. Defects of ER glycosyltransferases involved in the assembly of the lipid-linked oligosaccharide $\mathrm{GlcNAc}_{2} \mathrm{Man}_{9} \mathrm{Glc}_{3}$ limit the availability of this substrate for transfer to $\mathrm{N}$-glycosylation sites on acceptor proteins during translation (Figure 2). Such defects of $\mathrm{N}$-glycosylation lead to glycoproteins lacking whole N-glycan chains. Depending on the glycoproteins affected, nonoccupancy of $\mathrm{N}$-glycosylation sites can impair protein folding, secretion and stability. At the level of the organism, such defects lead to multiple organ dysfunctions. Neurological symptoms are frequent, including psychomotor retardation, ataxia, and hypotonia. Liver and cardiac dysfunctions are also frequently observed as well as endocrine disorders, which mainly affect the sexual maturation of female patients [14].

The functional impairments associated with some glycosyltransferase deficiencies reflect the functional relevance of the involved glycoproteins. For example, O-mannosylation [15] is an essential modification of $\alpha$-dystroglycan that ensures proper interactions between the dystroglycan complex and proteins of the extracellular matrix [16]. Such interactions are essential for the integrity of 
muscular fibers, for the migration of neurons in the cortex, and for the retinal architecture [17]. Because $\alpha$-dystroglycan is the main carrier of O-mannose chains, the manifestations of $\mathrm{O}$ mannosylation disorders relate to $\alpha$-dystroglycan functions, and therefore encompass muscular degeneration, brain abnormality, and blindness. Clinically, these disorders belong to the congenital muscular dystrophies and are known as Walker-Warburg syndrome, Muscle-Eye-Brain disease, Fukuyama-type congenital muscular dystrophy, and Limb-girdle muscular dystrophy. The most severe cases are usually associated with mutations in the core mannosyltransferase genes POMT1 [18] and POMT2 [19] and in the $\beta 1-2$ GICNAc-transferase gene POMGNT1 [20] (Figure 2), but other gene defects also account for severe cases of Walker-Warburg syndrome and Muscle-Eye-Brain disease. To date, defects in 12 genes are known to cause congenital muscular dystrophies, although the functions of some of these genes are still unclear. For example, the FKTN and FKRP genes encode putative glycosyltransferases involved in O-mannosylation, but their exact substrate specificity and activity remain unknown [21].

Another form of O-linked glycosylation is characterized by the addition of fucose (Fuc) to serine and threonine in the context of the epidermal growth factor (EGF)-like domains and thrombospondin-1 (TSP1) domains. Typical acceptor proteins are members of the Notch family including the ligands Jagged and Delta-like, which are signaling proteins involved in morphogenetic processes [22]. Complete deficiency of core O-fucosyltransferases POFUT1 and POFUT2 has not been described yet, but heterozygous mutations in the POFUT1 gene have been identified in cases of Dowling-Degos disease, an autosomal dominant pigmentation disorder [23]. Furthermore, mutations in the downstream acting glycosyltransferases, that is, the $\beta 1-3$ GlcNAc-transferase LFNG and the $\beta 1-3$ Glctransferase B3GALTL (Figure 2), have been associated with disorders of vertebral segmentation [24] and to multiple developmental defects known as Peters-Plus syndrome [25], respectively.

In general, defects of core glycosyltransferases are more severe than defects of terminal glycosylation. Nevertheless, the severity of the disease and the scope of organ involvements are also influenced by the functional redundancy inherent to specific glycosyltransferase reactions in the biosynthesis of classes of glycosylation. For example, mucin-type O-glycosylation is initiated by a large family of polypeptide GalNAc-transferases [26]. The partial redundancy in this enzyme family prevents a major loss of this type of glycans in humans, which explains why there is only one known disease of mucin-type O-glycosylation, called familial tumoral calcinosis, which is associated with mutations in the polypeptide GalNAc-transferase GALNT3 gene [27]. The hormone FGF23 requires for its secretion O-GalNAc glycans specifically added by GALNT3 in the Golgi apparatus [28]. Loss of FGF23 secretion leads to hyperphosphatemia and tissue calcification, which are the cardinal symptoms of tumoral calcinosis.

\section{Donor substrates}

Despite the hundreds of glycosyltransferases expressed in human cells, only eleven building blocks are used as donor substrates for the assembly of all human glycans. These substrates include nine nucleotide-activated sugars and two dolichol-phosphate (P) linked sugars (Figure 3A). Donor substrates are biosynthesized in the cytosol, or in the nucleus in the case of CMP-sialic acid (Sia), through multiple steps including interconversion between monosaccharide isomers. Donor substrates are used across classes of glycosylation, meaning that defects in the formation of individual nucleotide-activated sugars have a broad impact on glycan structures and lead to severe multiorgan disorders. However, the clinical severity of a given gene defect widely varies based on the 
level of residual activity enabled by individual mutations. For example, about 100 mutations have been described for the phosphomannomutase PMM2 gene [29], which represents by far the most frequent form of CDG. Mutations completely abrogating PMM2 activity lead to embryonic lethality [30] whereas point mutations that have minimal impact on the enzymatic activity will only cause mild intellectual disabilities. PMM2 activity mediates the conversion of mannose (Man)-6-P to Man-1-P, which is an early step in the biosynthesis of GDP-Man (Figure 3B). GDP-Man is further converted to dolichol-P-Man by an enzymatic complex encoded by the DPM1, DPM2, and DPM3 genes. Dolichol-PMan is a substrate used in $\mathrm{N}$-glycosylation, O-mannosylation, and for the biosynthesis of the glycosylphosphatidylinositol (GPI) anchors. Accordingly, decreased dolichol-P-Man availability causes of range of diseases sharing features of classical $\mathrm{N}$-glycosylation disorders, but also of the congenital muscular dystrophies caused by O-mannosylation defects.

Sometimes, exome sequencing of untyped CDG cases reveals mutations in genes that were previously associated with diseases unrelated to glycosylation. For example, phosphoglucomutase deficiency resulting from mutations in the PGM1 gene causes glycogen storage disease XIV, characterized by accumulation of glycogen in muscles because of reduced formation of Glc-6-P from Glc-1-P occurring during breakdown of glycogen [31]. The reverse reaction catalyzed by PGM1 (i.e., the formation of Glc-1-P from Glc-6-P) is also important for the subsequent formation of UDP-Gal, which is utilized for glycan formation (Figure 3B). Indeed, mutations in PGM1 have been identified as causing CDG with multiple clinical involvements such as growth retardation, cleft palate, muscular and cardiac disorders, and liver dysfunction among other manifestations [32].

The study of glycosylation diseases occasionally points to unexpected findings relative to the biological importance of donor substrate biosynthesis. The GNE gene encodes the bifunctional enzyme UDP-GIcNAc 2-epimerase/ManNAc kinase, which catalyzes a rate-limiting step in the biosynthesis of Sia [33]. The disruption of the Gne gene in mice is embryonic lethal [34], but decreased GNE activity in human beings is mainly associated with adult-onset, progressive limbgirdle muscle weakness with a remarkable sparing of quadriceps muscles [35]. This rather mild disease suggests that Sia can be efficiently salvaged in humans to bypass any defect of biosynthesis.

Because the biosynthetic pathways of most donor substrates are interconnected, it is tempting to try to therapeutically circumvent specific defects by increasing the supply of alternative carbohydrates that can be converted to the missing substrate. Unfortunately, such an approach has only been successful to treat the deficiency of Man-P isomerase (MPI), which catalyzes the interconversion of fructose-6-P and Man-6-P (Figure 3B). MPI deficiency is mainly a hepatic-intestinal disease and thus lacks the neurological involvement often found in CDG [36]. The decreased formation of Man-6-P accompanying MPI deficiency can be efficiently compensated by dietary Man supplementation, thereby alleviating disease symptoms [37]. Similarly, dietary supplementation with Gal has recently been shown to normalize serum transferrin glycosylation in patients affected of PGM1 deficiency [32], suggesting that Gal supplementation may alleviate some of the defects associated with the disease.

\section{Localization of donor substrates}

Nucleotide-activated sugars are synthesized in the cytosol and nucleus, but need to be transported to the lumen of the ER and Golgi apparatus for glycosylation reactions. Dedicated antiporters mediate the coupled translocation of nucleotide-activated sugars into the organelles and the return 
of corresponding nucleotide-monophosphates into the cytosol (Figure 1). Most antiporters are specific for a given nucleotide-activated sugar, although multi-specific transporters have also been described. For example, SLC35D1 transports UDP-GICA and UDP-GalNAc into the ER in exchange for returning UMP to the cytosol. These two nucleotide-activated sugars are involved in the biosynthesis of chondroitin sulfate, a main component of proteoglycans secreted by chondrocytes. Mutations in the SLC35D1 gene cause a skeletal disease called Schneckenbecken dysplasia, characterized by severe bone abnormalites leading to neonatal lethality [38]. It is likely that other classes of glycosylation are affected by the decreased transport of UDP-GIcA and UDP-GaINAc, but the extent of such alterations has not been addressed yet.

Additional defects of nucleotide-activated sugar transport have been associated with diseases, the symptoms of which reflect the importance of the implied carbohydrate for specific cellular functions. Mutations in the SLC35C1 gene encoding a Golgi GDP-Fuc transporter impairs terminal fucosylation, which yields epitopes such as $A B O$ and Lewis blood group antigens [39]. Some of these fucosylated epitopes function as ligands for selectins [40] and thereby participate to leukocyte adhesion and extravasation reactions [41]. Accordingly, the shortage of GDP-Fuc in the Golgi caused by defective transport impairs leukocyte trafficking and leads to increased bacterial infections. In addition, affected patients present with short stature, intellectual disability, and mild facial dysmorphism. Hematologic defects and susceptibility to infections were reverted by oral supplementation with Fuc [42]. By comparison, mutations in the CMP-Sia transporter gene SLC35A1 were found in a patient with intellectual impairment, seizures, ataxia, thrombocytopenia, renal and cardiac disorders [43]. A general conclusion about the role of sialylation cannot be drawn from these two cases, but the symptoms confirm the importance of Sia for leukocyte and platelet functions.

Dolichol-linked substrates do not use dedicated transporters to reach the ER lumen but proteins have been described that facilitate the translocation of these substrates across membranes. The first of these proteins is called MPDU1 and is required for making dolichol-P-Man and dolichol-P-Glc available to ER mannosyltransferases and glucosyltransferases [44]. These enzymes mediate the elongation of the dolichol-PP-oligosaccharide substrate for $\mathrm{N}$-glycosylation and participate in Omannosylation and GPI anchor biosynthesis. The mechanism of MPDU1 action is still unknown, but mutations in the MPDU1 gene lead to a form of CDG featuring symptoms typical of $\mathrm{N}$-glycosylation disorders, including psychomotor disability, hypotonia, and seizures $[45,46]$. Symptoms typical of CDG were also observed in patients harboring mutations in the RFT1 gene [47], which encodes a protein involved in the translocation of the precursor dolichol-PP-linked $\mathrm{GlCNAC}_{2} \mathrm{Man}_{5}$ from the cytosolic to the luminal side of the ER membrane [48] (Figure 2). Defective RFT1 activity results in the accumulation of dolichol-PP-GIcNAc2 $\mathrm{Man}_{5}$, which remains unavailable for further extension by luminally-oriented ER mannosyl- and glucosyltransferases.

\section{Localization of glycosyltransferases}

A precise localization of glycosyltransferases is also required for proper glycan maturation in the Golgi apparatus. Some glycosyltransferases concentrate in the cisternae of the cis-Golgi, whereas others accumulate in the trans-Golgi. The mechanisms underlying the distribution of glycosyltransferases are not completely understood, but proteins regulating vesicle transport are involved in the process. The Conserved Oligomeric Golgi (COG) complex orchestrates the recycling of medial- and cis-Golgi resident proteins by acting as a tether to connect COPI vesicles with cis-Golgi membranes [49]. COG defects lead to abnormal glycosylation [50] because of missorting of 
glycosylation enzymes and sugar transporters [51]. Whereas multiple classes of glycosylation are impaired, COG-related disorders are usually identified by detection of underglycosylated serum transferrin just like defects of N-glycosylation.

To date, mutations in seven out of eight COG subunit genes have been described. The most severe diseases are observed for COG6, COG7 and COG8 mutations, associated with severe neurological impairment, liver dysfunction, and infantile lethality [52-55]. The identification of milder cases of COG6 and COG7 deficiency harboring different mutations $[56,57]$ however shows that the severity of the disease does not simply relate to the subunit affected but rather to the capability of forming a fully functional COG complex. Besides the severe diseases observed for COG6 and COG7 defects, moderate clinical manifestations have been associated with mutations in COG1 [58, 59], COG2 [60], COG4 [61, 62], and COG5 [63-65].

COG subunits build a complex of two lobes, including COG1 to COG4 in lobe A and COG5 to COG8 in lobe B (Figure 4). In general, defects in lobe A lead to milder disease than defects in lobe B. Lobe A appears to be important for overall Golgi architecture, playing a role in Golgi organization and cisGolgi sorting [66]. Alterations in lobe A lead to accumulation of late glycosylation enzymes in COG complex vesicles, thereby preventing interaction with their substrate. Lobe $B$ rather mediates vesicular sorting of trans-Golgi enzymes through functional interactions with the tethering and fusion machinery of trans-Golgi cisternae $[67,68]$. Accordingly, glycosyltransferases from early Golgi cisternae, such as the $\beta 1$-2 GIcNAc-transferase MGAT1, are more affected by a defect in lobe A [66]. By contrast, Gal-transferases and Sia-transferases residing in trans-Golgi cisternae are more influenced by lobe B alterations [67]. Furthermore, lobe B deficiency mainly results in altered steady state levels of these enzymes due to their translocation to the ER and subsequent proteasomal degradation [65]. The broad involvement of lobe B in regulating glycosyltransferase and other transGolgi proteins probably account for the increased severity of lobe B mutations.

Whereas COG defects demonstrate the importance of glycosyltransferase localization for glycosylation, the characterization of another disease called Tn syndrome has pointed to the importance of chaperones in supporting folding and trafficking of specific glycosyltransferases. The Tn syndrome is a clonal defect of core $1 \beta 1-3$ Gal-transferase activity limited to a subset of hematopoietic cells. The presentation of bare O-linked GalNAc (the Tn antigen) on erythrocytes leads to the binding of naturally-occurring anti-Tn antibodies and hence to agglutination and hemolysis [69]. Tn antigen presentation on leukocytes and platelets may cause mild leukopenia and thrombocytopenia. Although core 1 1 1-3 Gal-transferase activity is decreased in Tn syndrome, no mutations have been found yet in the corresponding C1GALT1 gene. Rather, mutations in the COSMC gene encoding an ER-localized chaperone required for C1GALT1 folding have been identified as causing Tn syndrome [70]. C1GALT1 is the only glycosyltransferase known to undergo chaperoneassisted folding, but the example shows that proper glycosylation also relies on specific proteins such as COSMC that regulate the trafficking of glycosyltransferases from the ER to the Golgi.

\section{Organelle milieu}

Glycosyltransferases require co-factors, such as the metal ion $\mathrm{Mn}^{2+}$, and a range of environmental conditions to catalyze glycosylation reactions. The recent application of unbiased genetic approaches, such as homozygosity mapping and exome sequencing, has pointed to novel genes that affect glycosylation by regulating the acidification and ionic constituents of the secretory pathway. 
The ATP6VOA2 gene encodes a subunit of an $\mathrm{H}^{+}$-ATPase proton pump localized in the Golgi apparatus [71], which likely regulates $\mathrm{pH}$ in Golgi cisternae. Defective ATP6VOA2 action yields structural alterations of Golgi architecture but also causes accumulation of abnormal intracellular vesicles [72]. These changes affect multiple classes of glycosylation as shown by the abnormal N-glycosylation and mucin type O-glycosylation of blood serum proteins. Clinically, mutations in ATP6VOA2 lead to multiple abnormalities including growth delay and psychomotor disability, but also to skin wrinkling and connective tissue alterations referred to as cutis laxa [73]. Skin and skeletal phenotypes are likely related to alterations of extracellular matrix secretion as indicated by changes of TGF- $\beta$ signaling observed in affected fibroblasts [71].

Homozygosity mapping and exome sequencing also revealed mutations in the TMEM165 gene as causing a glycosylation disorder with broad clinical involvement. The five patients identified to date present with growth and developmental delay, hypotonia, skeletal abnormalities, and hepatomegaly [74]. TMEM165 is a transmembrane protein localized in the Golgi membrane but also found in the plasma membrane, late endosomes and lysosomes. The function of TMEM165 is unclear but appears to be related to the transport of calcium [75], which is normally found in high concentrations in the Golgi apparatus [76]. It is possible, but not yet tested, that TMEM165 dysfunctions might also affect $\mathrm{Mn}^{2+}$ import mediated by the SPCA1 $\mathrm{Ca}^{2+}$ pump [77], which would explain the broad glycosylation defect observed in TMEM165 patients.

Exome sequencing will continue to unravel genes of previously unknown or unclear function as causes of CDG. This growing catalog of gene defects will broaden our knowledge of the factors regulating glycosylation, but it will also bring forward new questions regarding the underlying mechanisms of such regulatory pathways. The recent characterization of TMEM165 is a good example outlining the difficult path following the description of mutations in a new gene. This work shows that biochemical and cell biological investigations are always required to understand the biological impact of genetic alterations.

\section{Concluding remarks}

This brief review of CDG illustrates the diversity of glycan functions by outlining the widespread consequences of alterations at specific points along biosynthetic pathways. While fascinating, the complexity of CDG and the broad range of disease severity within a CDG type, renders CDG diagnosis challenging. This suggests that CDG is probably underdiagnosed. Accordingly, the application of unbiased sequencing approaches will certainly reveal further gene defects as cause of CDG, but also unravel glycosylation defects in mild disorders such as non-syndromic intellectual disability [78]. Looking back at the evolving CDG landscape of the past 20 years, it has become clear that the description of these diseases has greatly increased the awareness of the biomedical community for the significance of glycosylation in human development and physiology.

\section{TEXT BOX: Glycosaminoglycans}

Disorders of glycosylation are not limited to altered glycosyltransferase activity and localization, donor substrate biosynthesis and translocation, and homeostasis of secretory organelles. After assembly of glycan chains, several carbohydrates are further modified by methylation, acylation, phosphorylation, and sulfation, thereby granting additional properties to glycoconjugates. 
Sulfation is for example a prominent modification found on glycosaminoglycans [79], which decorate proteoglycans and other extracellular matrix proteins. Glycosaminoglycans are linear chains featuring repeats of disaccharide motifs, which defines subclasses named heparan sulfate, chondroitin sulfate, dermatan sulfate, and keratan sulfate. The extensive sulfation of glycosaminoglycans increases water retention, thereby contributing to tissue hydration and swelling. In addition to affecting physical properties, the arrangement of sulfated groups on glycosaminoglycan chains generate specific binding sites for soluble proteins, such as growth factors, cytokines, and coagulation enzymes. Through this binding of growth and differentiation factors, glycosaminoglycans are essential for the formation of morphogenetic gradients [80], which direct organ formation during embryogenesis and postnatal tissue growth. Accordingly, general alterations of glycosaminoglycan biosynthesis are embryonic lethal. Despite their essential functions during embryogenesis, eight genetic defects of glycosaminoglycan assembly have been identified to date. These defects consists of the genes XYLT1, $B 3 G A L T 7, B 3 G A L T 6, B 3 G A T 3$ encoding glycosyltransferases building the tetrasaccharide core $X y l(\beta 1$ 4)Gal( $(\beta 1-3) G a l(\beta 1-3) G l c A$, the polymerizing glycosyltransferase EXT1, EXT2, and CHSY1 genes, and the dermatan sulfate epimerase DSE gene [81]. These defects of glycosaminoglycan biosynthesis are associated with skeletal and connective tissue disorders such as multiple hereditary exostoses, Desbuquois dysplasia, and different forms of the Ehlers-Danlos syndrome.

Alterations of glycosaminoglycan sulfation also cause different connective tissue disorders. To date, mutations in the three CHST3 [82], CHST6 [83], and CHST14 [84] sulfotransferase genes have been recognized in cases of spondyloepiphyseal dysplasia, macular corneal dystrophy, and Ehlers-Danlos syndrome musculocontractural type 1 , respectively. The CHST3 sulfotransferase catalyzes the 6-Osulfation of GalNAc in chondroitin and dermatan sulfate, whereas CHST6 catalyzes the 6-O-sulfation of GICNAc in keratin sulfate, and CHST14 the 4-O-sulfation of GalNAc in dermatan sulfate. Limited availability of the sulfotransferase donor substrate 3'-phosphoadenosine-5'-phosphosulfate caused by mutations in the PAPSS2 synthase gene also lead to a skeletal disease [85], which shares phenotypic similarities with several defects of glycosaminoglycan biosynthesis and sulfate.

\section{Acknowledgements}

We thank Eric Berger for his valuable comments. This work was supported by the Swiss National Foundation grant 310030-149949 to T. Hennet. 


\section{References}

1 Cornfield, A. and Berry, M. (2015) Current aspects of eukaryotic glycosylation. Trends Biochem Sci in press

2 Turnbull, J.E. (2015) Complexity and functional diversity of glycosaminoglycans: master cell regulators. Trends Biochem Sci in press

3 Schengrund, C.L. (2015) Gangliosides: glycosphingolipids essential for normal neural development and function. Trends Biochem Sci in press

4 Ledeen, R.W. and Wu, G. (2015) The multi-tasked life of ganglioside GM1, a true factotum of nature. Trends Biochem Sci in press

5 Moremen, K.W. et al. (2012) Vertebrate protein glycosylation: diversity, synthesis and function. Nat Rev Mol Cell Biol 13, 448-462

6 Babovic-Vuksanovic, D. and O'Brien, J.F. (2007) Laboratory diagnosis of congenital disorders of glycosylation type I by analysis of transferrin glycoforms. Mol Diagn Ther 11, 303-311

7 Matthijs, G. et al. (2013) Approaches to homozygosity mapping and exome sequencing for the identification of novel types of CDG. Glycoconj J 30, 67-76

8 Hennet, T. (2012) Diseases of glycosylation beyond classical congenital disorders of glycosylation. Biochim Biophys Acta 1820, 1306-1317

$9 \quad$ Freeze, H.H. et al. (2014) Solving Glycosylation Disorders: Fundamental Approaches Reveal Complicated Pathways. Am J Hum Genet 94, 161-175

10 Aebi, M. et al. (1999) Carbohydrate-deficient glycoprotein syndromes become congenital disorders of glycosylation: an updated nomenclature for CDG. First International Workshop on CDGS. Glycoconj.J. 16, 669-671

11 Jaeken, J. et al. (2009) CDG nomenclature: time for a change! Biochim Biophys Acta 1792, 825-826

12 Varki, A. and Marth, J.D. (1995) Oligosaccharides in vertebrate development. Seminars in Developmental Biology 6, 127-138

13 Breton, C. et al. (2012) Recent structures, evolution and mechanisms of glycosyltransferases. Curr Opin Struct Biol 22, 540-549

14 de Zegher, F. and Jaeken, J. (1995) Endocrinology of the carbohydrate-deficient glycoprotein syndrome type 1 from birth through adolescence. Pediatr.Res. 37, 395-401

15 Loibl, M. and Strahl, S. (2013) Protein O-mannosylation: what we have learned from baker's yeast. Biochim Biophys Acta 1833, 2438-2446

16 Godfrey, C. et al. (2011) Dystroglycanopathies: coming into focus. Curr Opin Genet Dev 21, 278-285

17 Abad-Rodriguez, J. and Diez-Revuelta, N. (2015) Axon glycoprotein routing in nerve polarity, function and repair. Trends Biochem Sci in press 
18 Beltran-Valero de Bernabe, D. et al. (2002) Mutations in the O-mannosyltransferase gene POMT1 give rise to the severe neuronal migration disorder Walker-Warburg syndrome. Am J Hum Genet 71, 1033-1043

19 van Reeuwijk, J. et al. (2005) POMT2 mutations cause alpha-dystroglycan hypoglycosylation and Walker Warburg syndrome. J Med Genet 42, 907-912

20 Yoshida, A. et al. (2001) Muscular dystrophy and neuronal migration disorder caused by mutations in a glycosyltransferase, POMGnT1. Dev.Cell 1, 717-724

21 Praissman, J.L. and Wells, L. (2014) Mammalian O-mannosylation pathway: glycan structures, enzymes, and protein substrates. Biochemistry 53, 3066-3078

22 Stanley, P. and Okajima, T. (2010) Roles of glycosylation in Notch signaling. Curr Top Dev Biol 92, 131-164

$23 \mathrm{Li}$, M. et al. (2013) Mutations in POFUT1, encoding protein O-fucosyltransferase 1, cause generalized Dowling-Degos disease. Am J Hum Genet 92, 895-903

24 Sparrow, D.B. et al. (2006) Mutation of the lunatic fringe gene in humans causes spondylocostal dysostosis with a severe vertebral phenotype. Am J Hum Genet 78, 28-37

25 Lesnik Oberstein, S.A. et al. (2006) Peters Plus syndrome is caused by mutations in B3GALTL, a putative glycosyltransferase. Am J Hum Genet 79, 562-566

26 Bennett, E.P. et al. (2012) Control of mucin-type O-glycosylation: a classification of the polypeptide GalNAc-transferase gene family. Glycobiology 22, 736-756

27 Topaz, O. et al. (2004) Mutations in GALNT3, encoding a protein involved in O-linked glycosylation, cause familial tumoral calcinosis. Nat Genet 36, 579-581

28 Kato, K. et al. (2006) Polypeptide GalNAc-transferase T3 and familial tumoral calcinosis. Secretion of fibroblast growth factor 23 requires O-glycosylation. J Biol Chem 281, 18370 18377

29 Haeuptle, M.A. and Hennet, T. (2009) Congenital disorders of glycosylation: an update on defects affecting the biosynthesis of dolichol-linked oligosaccharides. Human Mutation in press

30 Thiel, C. et al. (2006) Targeted disruption of the mouse phosphomannomutase 2 gene causes early embryonic lethality. Mol Cell Biol 26, 5615-5620

31 Stojkovic, T. et al. (2009) Muscle glycogenosis due to phosphoglucomutase 1 deficiency. $N$ Engl J Med 361, 425-427

32 Tegtmeyer, L.C. et al. (2014) Multiple phenotypes in phosphoglucomutase 1 deficiency. $N$ Engl J Med 370, 533-542

33 Keppler, O.T. et al. (1999) UDP-GIcNAc 2-epimerase: a regulator of cell surface sialylation. Science 284, 1372-1376

34 Schwarzkopf, M. et al. (2002) Sialylation is essential for early development in mice. Proc Natl Acad Sci U S A 99, 5267-5270 
Eisenberg, I. et al. (2001) The UDP-N-acetylglucosamine 2-epimerase/N-acetylmannosamine kinase gene is mutated in recessive hereditary inclusion body myopathy. Nat.Genet. $29,83-$ 87

36 Pelletier, V.A. et al. (1986) Secretory diarrhea with protein-losing enteropathy, enterocolitis cystica superficialis, intestinal lymphangiectasia, and congenital hepatic fibrosis: a new syndrome. J Pediatr 108, 61-65

37 Niehues, R. et al. (1998) Carbohydrate-deficient glycoprotein syndrome type lb. Phosphomannose isomerase deficiency and mannose therapy. J.Clin.Invest. 101, 1414-1420

38 Hiraoka, S. et al. (2007) Nucleotide-sugar transporter SLC35D1 is critical to chondroitin sulfate synthesis in cartilage and skeletal development in mouse and human. Nat Med 13, 1363-1367

39 Lubke, T. et al. (2001) Complementation cloning identifies CDG-IIc, a new type of congenital disorders of glycosylation, as a GDP-fucose transporter deficiency. Nat.Genet. 28, 73-76

40 Gabius, H.J. (2015) The glycobiology of the CD system: a dictionary for translating marker designations into glycan/lectin structure and function. Trends Biochem Sci in press

41 Zarbock, A. et al. (2011) Leukocyte ligands for endothelial selectins: specialized glycoconjugates that mediate rolling and signaling under flow. Blood 118, 6743-6751

42 Marquardt, T. et al. (1999) Correction of leukocyte adhesion deficiency type II with oral fucose. Blood 94, 3976-3985

43 Mohamed, M. et al. (2013) Intellectual disability and bleeding diathesis due to deficient CMP-sialic acid transport. Neurology 81, 681-687

44 Ware, F.E. and Lehrman, M.A. (1996) Expression cloning of a novel suppressor of the Lec15 and Lec35 glycosylation mutations of Chinese hamster ovary cells. J.Biol.Chem. 271, 1393513938

45 Schenk, B. et al. (2001) MPDU1 mutations underlie a novel human congenital disorder of glycosylation (CDG), designated type If. J.Clin.Invest. 108, 1687-1695

46 Kranz, C. et al. (2001) A mutation in the human MPDU1 gene causes congenital disorder of glycosylation type If (CDG-If). J.Clin.Invest 108, 1613-1619

47 Haeuptle, M.A. et al. (2008) Human RFT1 deficiency leads to a disorder of N-linked glycosylation. Am J Hum Genet 82, 600-606

48 Helenius, J. et al. (2002) Translocation of lipid-linked oligosaccharides across the ER membrane requires Rft1 protein. Nature $415,447-450$

49 Ungar, D. et al. (2002) Characterization of a mammalian Golgi-localized protein complex, COG, that is required for normal Golgi morphology and function. J Cell Biol 157, 405-415

50 Kingsley, D.M. et al. (1986) Three types of low density lipoprotein receptor-deficient mutant have pleiotropic defects in the synthesis of $\mathrm{N}$-linked, O-linked, and lipid-linked carbohydrate chains. J Cell Biol 102, 1576-1585 
51 Oka, T. et al. (2004) The COG and COPI complexes interact to control the abundance of GEARs, a subset of Golgi integral membrane proteins. Mol Biol Cell 15, 2423-2435

$52 \mathrm{Wu}, \mathrm{X}$. et al. (2004) Mutation of the COG complex subunit gene COG7 causes a lethal congenital disorder. Nat Med 10, 518-523

53 Lubbehusen, J. et al. (2010) Fatal outcome due to deficiency of subunit 6 of the conserved oligomeric Golgi complex leading to a new type of congenital disorders of glycosylation. Hum Mol Genet 19, 3623-3633

54 Kranz, C. et al. (2007) COG8 deficiency causes new congenital disorder of glycosylation type IIh. Hum Mol Genet 16, 731-741

55 Foulquier, F. et al. (2007) A new inborn error of glycosylation due to a Cog8 deficiency reveals a critical role for the Cog1-Cog8 interaction in COG complex formation. Hum Mol Genet 16, 717-730

56 Huybrechts, S. et al. (2012) Deficiency of Subunit 6 of the Conserved Oligomeric Golgi Complex (COG6-CDG): Second Patient, Different Phenotype. JIMD Rep 4, 103-108

57 Zeevaert, R. et al. (2009) A new mutation in COG7 extends the spectrum of COG subunit deficiencies. Eur J Med Genet 52, 303-305

58 Foulquier, F. et al. (2006) Conserved oligomeric Golgi complex subunit 1 deficiency reveals a previously uncharacterized congenital disorder of glycosylation type II. Proc Natl Acad Sci U S A 103, 3764-3769

59 Zeevaert, R. et al. (2009) Cerebrocostomandibular-like syndrome and a mutation in the conserved oligomeric Golgi complex, subunit 1. Hum Mol Genet 18, 517-524

60 Kodera, H. et al. (2014) Mutations in COG2 encoding a subunit of the conserved oligomeric golgi complex cause a congenital disorder of glycosylation. Clinical genetics

61 Reynders, E. et al. (2009) Golgi function and dysfunction in the first COG4-deficient CDG type II patient. Hum Mol Genet 18, 3244-3256

62 Ng, B.G. et al. (2011) Identification of the first COG-CDG patient of Indian origin. Mol Genet Metab 102, 364-367

63 Paesold-Burda, P. et al. (2009) Deficiency in COG5 causes a moderate form of congenital disorders of glycosylation. Hum Mol Genet 18, 4350-4356

64 Fung, C.W. et al. (2012) COG5-CDG with a Mild Neurohepatic Presentation. JIMD Rep 3, 6770

65 Rymen, D. et al. (2012) COG5-CDG: expanding the clinical spectrum. Orphanet J Rare Dis 7, 94

66 Peanne, R. et al. (2011) Differential effects of lobe A and lobe B of the Conserved Oligomeric Golgi complex on the stability of \{beta\}1,4-galactosyltransferase 1 and \{alpha\}2,6-

sialyltransferase 1. Glycobiology 21, 864-876

67 Pokrovskaya, I.D. et al. (2011) Conserved oligomeric Golgi complex specifically regulates the maintenance of Golgi glycosylation machinery. Glycobiology 21, 1554-1569 
68 Willett, R. et al. (2013) COG complexes form spatial landmarks for distinct SNARE complexes. Nat Commun 4, 1553

69 Berger, E.G. (1999) Tn-syndrome. Biochim Biophys Acta 1455, 255-268

$70 \mathrm{Ju}, \mathrm{T}$. and Cummings, R.D. (2005) Protein glycosylation: chaperone mutation in Tn syndrome. Nature 437, 1252

71 Fischer, B. et al. (2012) Further characterization of ATP6V0A2-related autosomal recessive cutis laxa. Hum Genet 131, 1761-1773

72 Hucthagowder, V. et al. (2009) Loss-of-function mutations in ATP6V0A2 impair vesicular trafficking, tropoelastin secretion and cell survival. Hum Mol Genet 18, 2149-2165

73 Kornak, U. et al. (2008) Impaired glycosylation and cutis laxa caused by mutations in the vesicular H+-ATPase subunit ATP6VOA2. Nat Genet 40, 32-34

74 Foulquier, F. et al. (2012) TMEM165 deficiency causes a congenital disorder of glycosylation. Am J Hum Genet 91, 15-26

75 Demaegd, D. et al. (2013) Newly characterized Golgi-localized family of proteins is involved in calcium and pH homeostasis in yeast and human cells. Proc Natl Acad Sci U S A 110, 68596864

76 Pizzo, P. et al. (2010) The trans-golgi compartment: A new distinct intracellular Ca store. Commun Integr Biol 3, 462-464

77 Vanoevelen, J. et al. (2005) The secretory pathway Ca2+/Mn2+-ATPase 2 is a Golgi-localized pump with high affinity for Ca2+ ions. J Biol Chem 280, 22800-22808

78 Molinari, F. et al. (2008) Oligosaccharyltransferase-subunit mutations in nonsyndromic mental retardation. Am J Hum Genet 82, 1150-1157

79 Kusche-Gullberg, M. and Kjellen, L. (2003) Sulfotransferases in glycosaminoglycan biosynthesis. Curr Opin Struct Biol 13, 605-611

80 Hacker, U. et al. (2005) Heparan sulphate proteoglycans: the sweet side of development. Nat Rev Mol Cell Biol 6, 530-541

81 Mizumoto, S. et al. (2013) Human genetic disorders caused by mutations in genes encoding biosynthetic enzymes for sulfated glycosaminoglycans. J Biol Chem 288, 10953-10961

82 Thiele, H. et al. (2004) Loss of chondroitin 6-O-sulfotransferase-1 function results in severe human chondrodysplasia with progressive spinal involvement. Proc Natl Acad Sci U S A 101, 10155-10160

83 Akama, T.O. et al. (2000) Macular corneal dystrophy type I and type II are caused by distinct mutations in a new sulphotransferase gene. Nat Genet 26, 237-241

84 Dundar, M. et al. (2009) Loss of dermatan-4-sulfotransferase 1 function results in adducted thumb-clubfoot syndrome. Am J Hum Genet 85, 873-882

85 Faiyaz ul Haque, M. et al. (1998) Mutations in orthologous genes in human spondyloepimetaphyseal dysplasia and the brachymorphic mouse. Nat Genet 20, 157-162 


\section{Figure legends}

Figure 1. Glycosylation reaction. Schematic representation of the key players required for glycosylation reactions occurring in the Golgi apparatus. The biosynthesis of nucleotide-activated sugars (NDP-sugar) takes place in the cytosol whereas glycosyltransferase enzymes are localized on the luminal side of the endomembranes of the secretory pathway. Transporter systems are required for the import of nucleotide-activated sugars into the Golgi apparatus and for maintaining optimal ionic conditions in the organelle, thereby regulating $\mathrm{pH}, \mathrm{Mn}^{2+}$ import and $\mathrm{P}$ export.

Figure 2. Biosynthesis of core structures for $\mathrm{N}$-glycosylation, O-mannosylation, and O-fucosylation. Nglycosylation begins at the ER membrane by the stepwise assembly of dolichol-PP-GlcNAc $\mathrm{Man}_{9} \mathrm{Glc}_{3}$, which is transferred to the selected Asn residues of nascent glycoproteins by the oligosaccharyltransferase complex (OST). O-Mannosylated and O-fucosylated glycans are shaped by the sequential addition of different monosaccharides based on the acceptor specificity of glycosyltransferases.

Figure 3. Donor substrates of glycosyltransferases. A, List of donor substrates utilized in human cells for glycosylation reactions. The substrates are grouped by nucleotide types and dolichol-P (DolP). B, Biosynthesis pathways of UDP-Gal, UDP-GIc, DolP-GIc, GDP-Man, and DolP-Man. The positions of known gene defects are marked with the corresponding gene symbols. GALK1, galactokinase; GALT, Gal-1-P uridylyltransferase; GALE, UDP-Gal 4-epimerase; PGM1, phosphoglucomutase 1; MPI, Man-P isomerase; PMM2, phosphomannomutase 2; GMPPA/GMPPB, GDP-Man pyrophosphorylase A/B; DPM1/2/3, DolP-Man synthase 1/2/3.

Figure 4. Schematic organization of the Conserved Oligomeric Golgi (COG) complex with display of lobe contributions to organelle architecture and protein trafficking. The eight COG subunits form two lobes, designated A (blue) and B (red). Interactions between subunits of a lobe are indicated with triple and quadruple lines. Lobe $A$ and lobe B interact through COG1 and COG8 subunits (black line). Both lobes interact with COPI tether proteins (Golgin84, p115) and several SNARE proteins (STX5, STX6, Sly1, GS27, SNAP29), which are outlined in orange boxes. Lobe A and B mediate vesicular retrograde transport of cis (MGAT1, MAN2A1, ST3GAL5) and medial (B4GALT1, ST3GAL1, ST6GAL1) Golgi enzymes, respectively. Golgi glycosyltransferases are outlined in green boxes. 
Figure 1

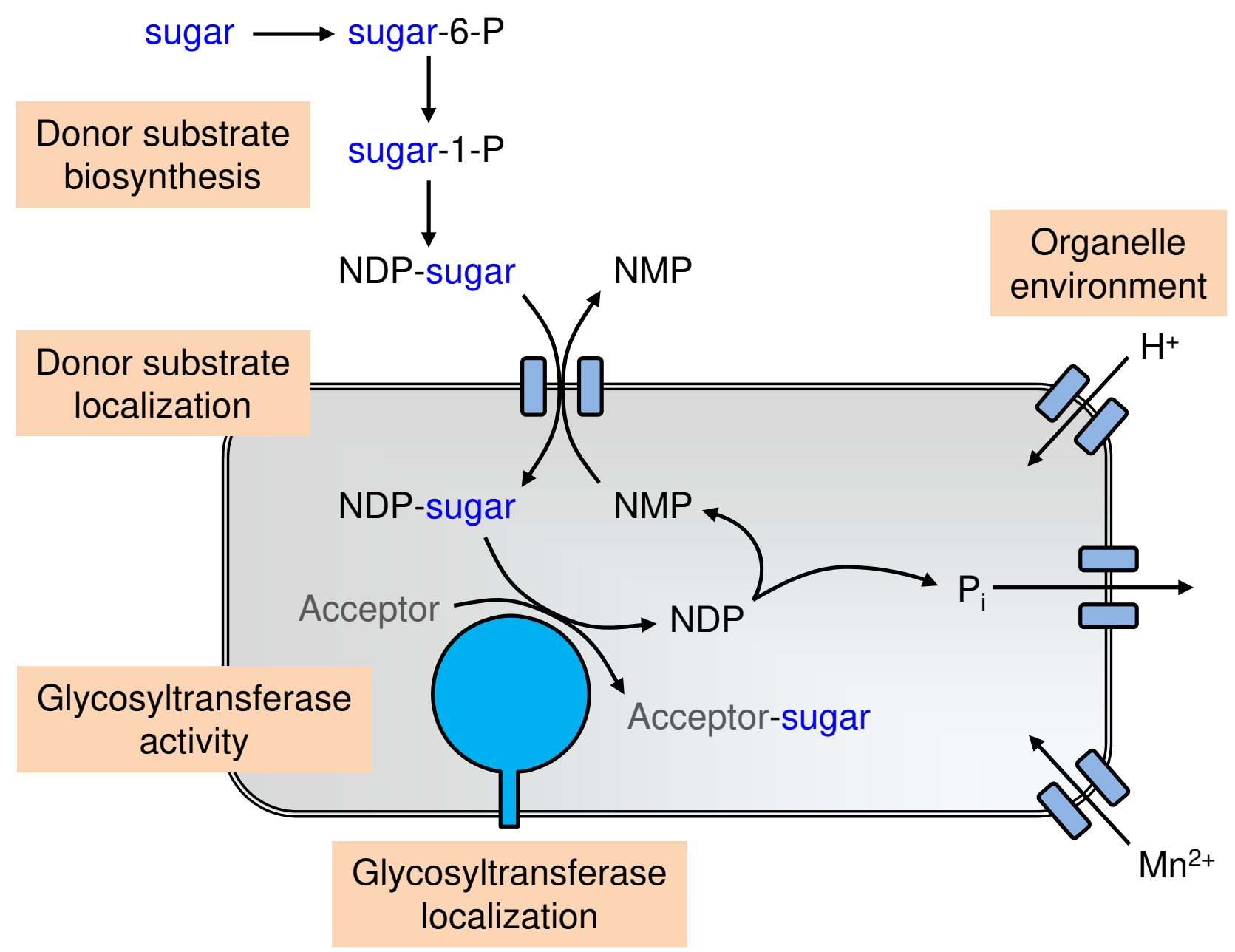


Figure 2

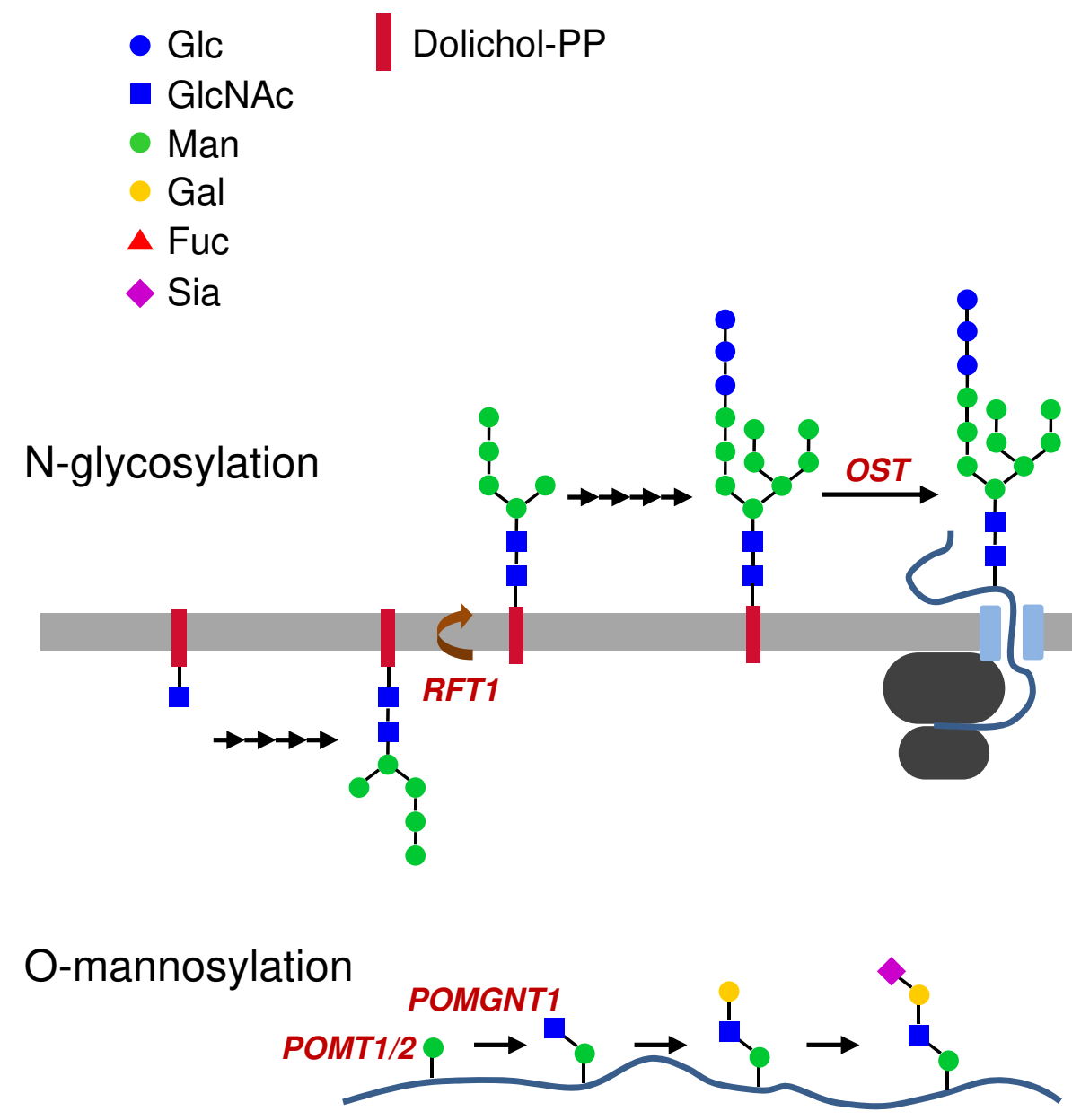

O-fucosylation
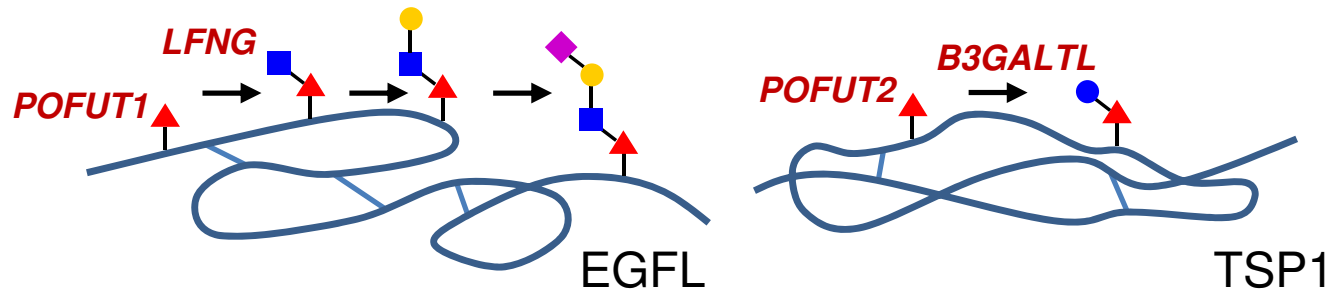
Figure 3

A

$\begin{array}{lll}\text { UDP-Glc } & \text { GDP-Man } & \text { DolP-Man } \\ \text { UDP-GlcNAc } & \text { GDP-Fuc } & \text { DolP-Glc } \\ \text { UDP-Gal } & & \\ \text { UDP-GalNAc } & \\ \text { UDP-GlcA } & \text { CMP-Sia } & \\ \text { UDP-Xyl } & & \end{array}$

B

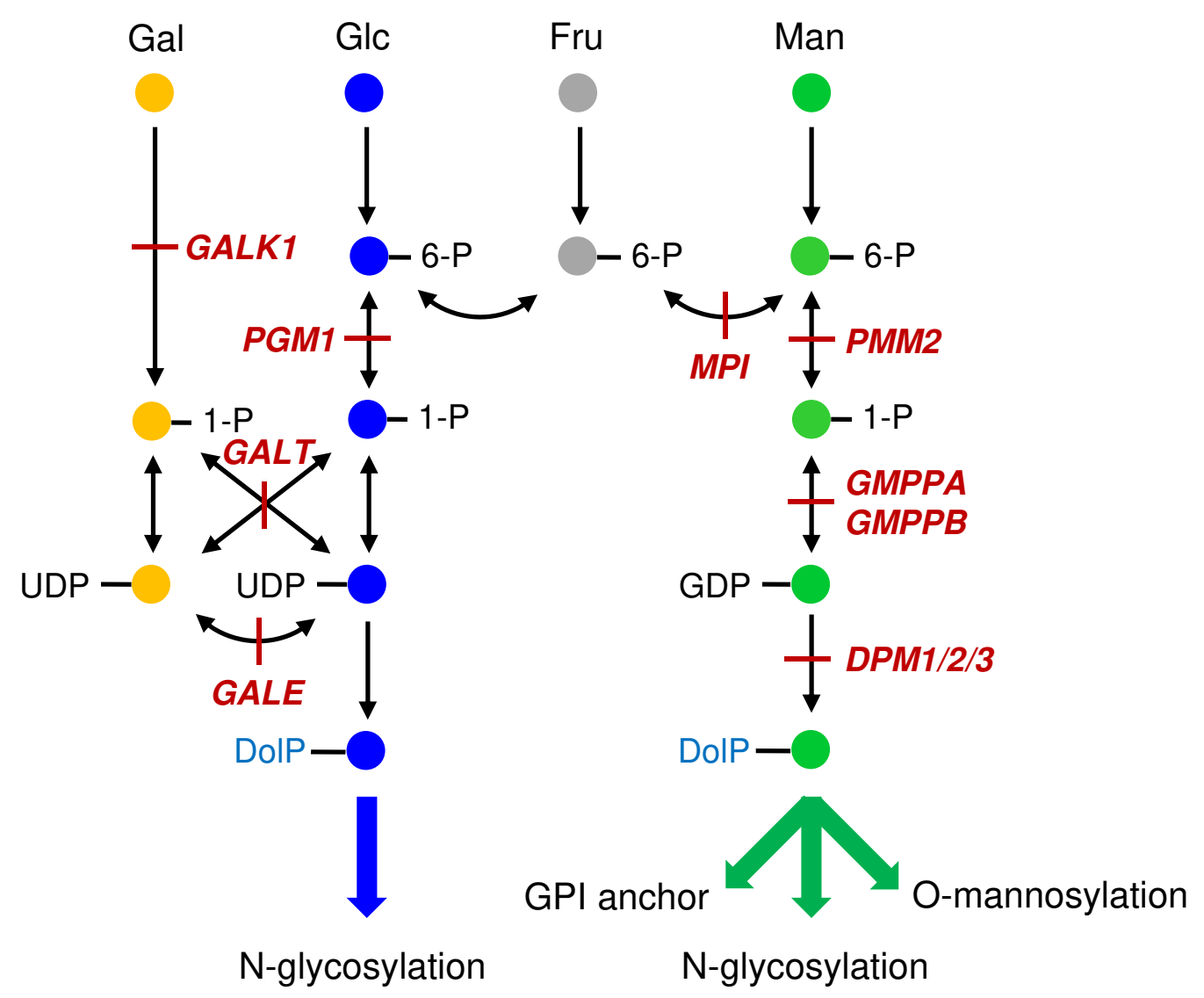


Figure 4

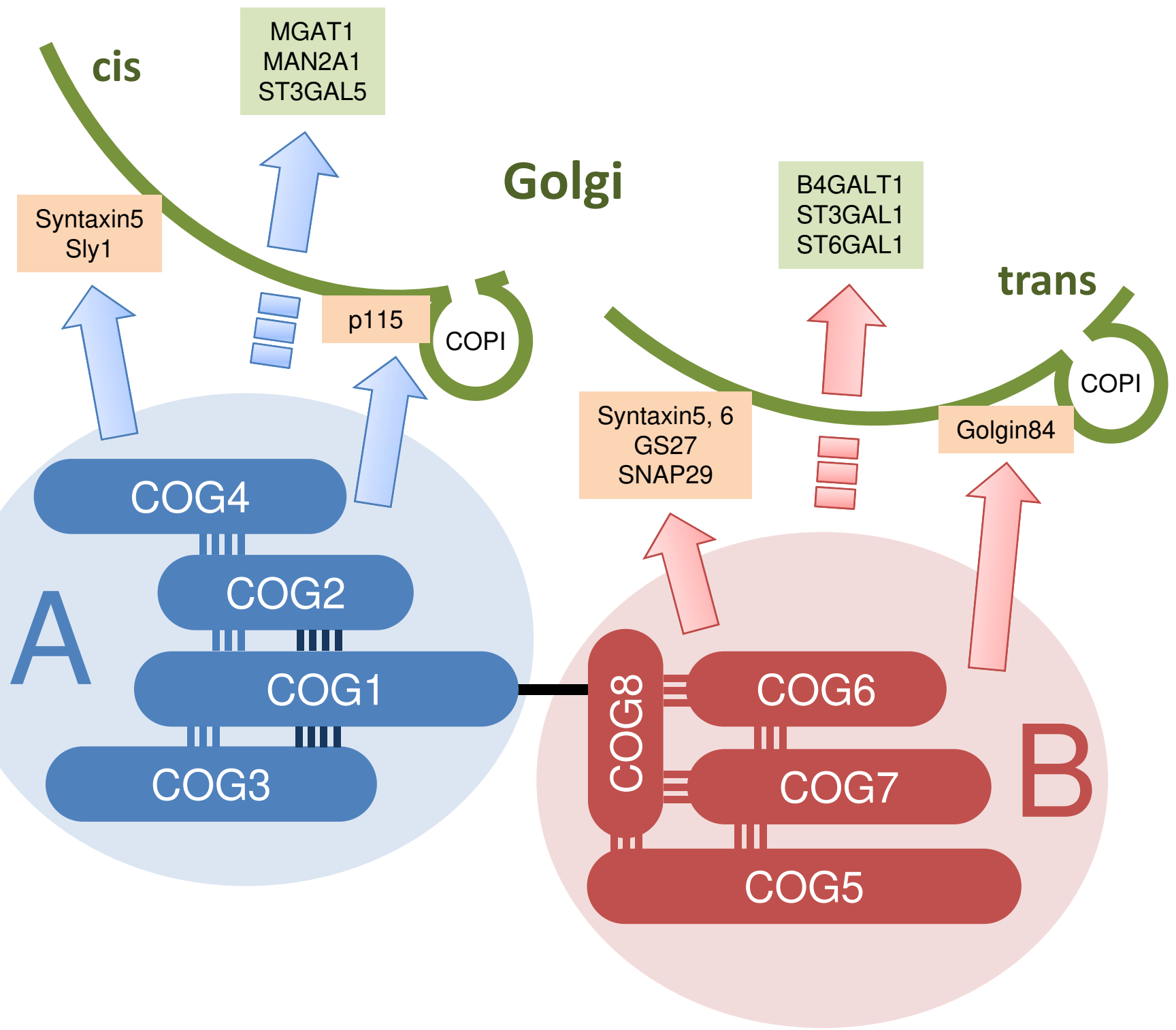

